\title{
Two Color Microarray
}

National Cancer Institute

\section{Source}

National Cancer Institute. Two Color Microarray. NCI Thesaurus. Code C116153.

A genomic microarray-based method using cDNA from two samples, labeled with two separate fluorophores/dyes at different emission waveleng ths, and hybridized to a single microarray for laser excitation in a microarray scanner. Relative fluorescence intensity of each dye can be measured and used in log- or intensity- ratio based comparative analysis for gene expression between the two experimental conditions. 